\title{
Studies on Regional Wealth Inequalities: The Case of Italy
}

\author{
M. Ausloos ${ }^{a, b, c, *}$ And R. Cerqueti ${ }^{d}$ \\ ${ }^{a}$ School of Management, University of Leicester, University Road, Leicester, LE1 7RH, UK \\ ${ }^{b}$ eHumanities group ${ }^{\dagger}$, Royal Netherlands Academy of Arts and Sciences, \\ Joan Muyskenweg 25, 1096 CJ Amsterdam, The Netherlands \\ ${ }^{c}$ Group of Researchers for Applications of Physics in Economy and Sociology, \\ rue de la Belle Jardiniere 483, B-4031, Angleur, Belgium \\ ${ }^{d}$ University of Macerata, Department of Economics and Law, via Crescimbeni 20, I-62100, Macerata, Italy \\ The paper contains a short review of techniques examining regional wealth inequalities based on recently \\ published research work, but also presenting unpublished features. The data pertains to Italy over the period \\ 2007-2011: the number of cities in regions, the number of inhabitants in cities and in regions, as well as the \\ aggregated tax income of the cities and of regions. Frequency-size plots and cumulative distribution function plots, \\ scatter plots and rank-size plots are displayed. The rank-size rule of a few cases is discussed. Yearly data of the \\ aggregated tax income is transformed into a few indicators: the Gini, Theil, and Herfindahl-Hirschman indices. \\ Numerical results confirm that IT is divided into very different regional realities. One region is selected for a short \\ discussion: Molise. A note on the "first digit Benford law" for testing data validity is presented.
}

DOI: 10.12693/APhysPolA.129.959

PACS/topics: 89.65.Gh, 93.30.-w, 05.20.-y, 05.10.Ln

\section{Introduction}

In studying geo-complexity, many variables come to mind. Beside geo-political aspects, many considerations are social, economic and financial ones. One is aware that local aspects should be distinguished from global ones. Various levels can be imagined for discussion and modeling. Here below, the paper contains a short review of techniques and results examining regional wealth inequalities based on recently published research work [16], but also presenting unpublished features. In brief, our aim is to find correlations between long lived macro-level and shorter lived micro-level features.

The data pertains to Italy (IT), over the quinquennium period 2007-2011: (i) the number of cities in regions, (ii) the number of inhabitants in cities and (iii) in regions, based on the 2011 Census, as well as (iv) the yearly aggregated tax income of the cities and (v) of regions, from 2007 till 2011, but the ideas can be carried forward to other countries or systems. Rank-size plots and cumulative distribution function plots are displayed in Sect. 2. Frequency size and scatter plots are to be found in the listed references.

In Sect. 3, the yearly data of the aggregated tax income (ATI), i.e. the city contribution to the national GDP from citizens income tax, are explored through a rank-size analysis. In Sect. 4 financial inequalities are examined: the ATI data is transformed into a few usual indicators of wealth distributions: the Theil index [7], a HerfindahlHirschman index [8] and the Gini coefficient [9].

\footnotetext{
*corresponding author; e-mail: ma683@le.ac.uk

${ }^{\dagger}$ Associate Researcher
}

One region has been selected for illustrating a smaller entity than the entire country, i.e. the Molise region. In order not to distract from the aim, a consideration on data validity, with emphasis on Molise and IT is given at the end through Benford law first digit [10, 11]; see Sect. 5.

\section{Administrative distribution of cities in regions}

As far as 2011, Italy is composed of 20 regions, 110 provinces and 8092 municipalities, respectively. Since 2007, the number of IT cities has been yearly evolving to become respectively: $8101,8094,8094,8092,8092$, from 2007 till 2011.

In brief, several cities have thus merged into new ones, other were phagocytized. To sum up: 13 cities became 4 ones in two steps, over the studied quinquennium. For completeness, let us mention them: Campolongo Tapogliano (UD), Ledro (TN), Comano Terme (TN), and Gravedona ed Uniti (CO). Moreover, a few (7) cities changed both province and region membership: such 7 cities moved from PU (the province of Pesaro and Urbino) in the Marche region, to RN (province of Rimini) in the Emilia Romagna region (Casteldelci, Maiolo, Novafeltria, Pennabilli, San Leo, Sant' Agata Feltria, Talamello). For completeness, notice that 228 municipalities have changed from a province to another one, but nevertheless remained in the same region.

For completeness, let it be mentioned that in 2007, the number of provinces was increased from 103 (to 110) by 7 units (BT, CI, FM, MB, OG, OT, VS)* thereafter,

\footnotetext{
*see ISO code:

http://en.wikipedia.org/wiki/Provinces_of_Italy.
} 
leading to 110 provinces; 4 provinces have been instituted by a regional law of 12 July 2001 in Sardinia and became operative in 2005 (CI MB, OG, OT), while BT, FM and VS have been created on June 11th, 2004 and became operative on June 2009.

The number of regions has remained constantly equal to 20 since 1963 . It should be here mentioned that Molise (MOL) is the youngest Italian region, being established in 1963, when the region "Abruzzi e Molise" was split. Campobasso (CB) and Isernia (IS) are the Italian provinces of Molise.

For further historical and data details, we address the reader to: http://www.comuni-italiani.it/regioni.html. The above indicates that some care had to be taken to compare entities at various times in the quinquennium, i.e. when necessary "data manipulation through summation" occurred.

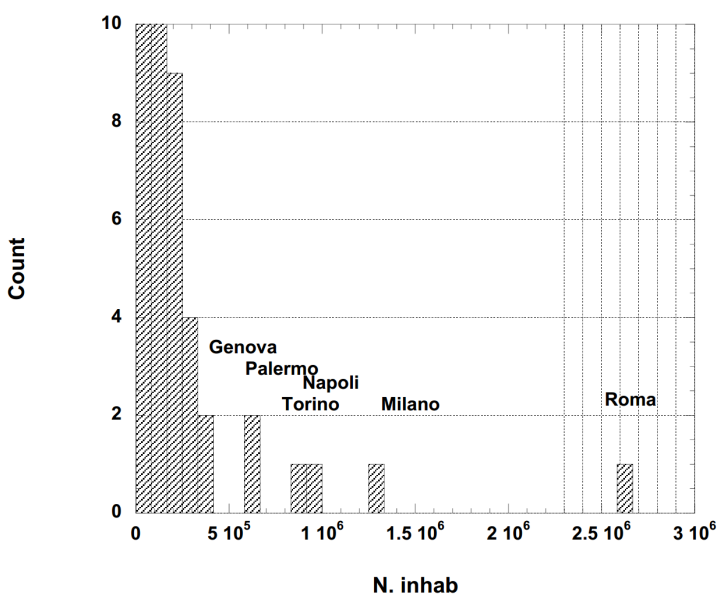

Fig. 1. Histogram of the number of large cities in IT having a population of $N$ inhabitants; the $y$-axis is truncated at a value $=10$ for readability in order to emphasize the 6 main cities.

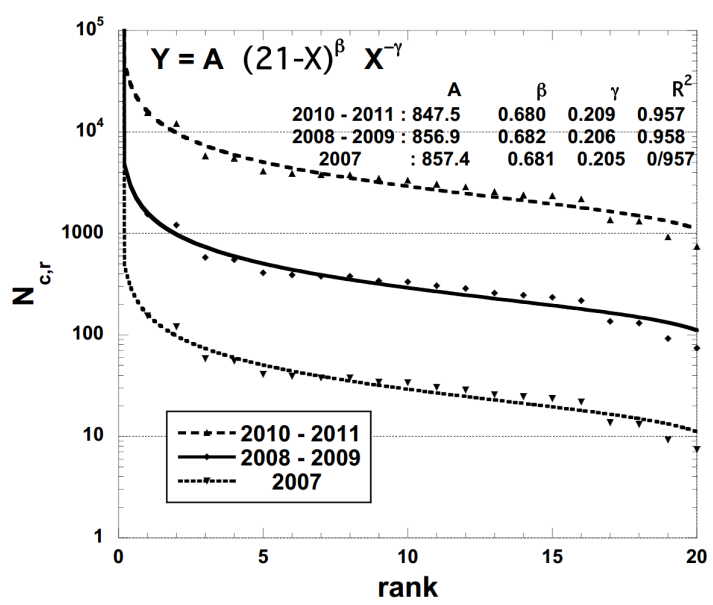

Fig. 2. Display of the rank-size law of the number $\left(N_{c, r}\right)$ of cities in a given region for the IT regions $(N=20)$ in various recent years; data for 2007 and for 2010-2011 are displaced for readability; fit values for a 3-parameter function, as Eq. (2.1), are given for the undisplaced data.
Let us illustrate the global view of IT cities through a histogram presenting (counting) the number of cities having a population of $N$ inhabitants, as in Fig. 1, according to the 2011 Census. In order to emphasize the 6 main cities, the $y$-axis is truncated at a value $=10$ for readability. It can be shown that these 6 cities are outliers in subsequent statistical analysis of all pertinent data. In so doing, the notion of King $(\mathrm{K})$ and that of Vice-Roy (VR) cities can be introduced [2, 12, 13]. Such outliers always occur, at low rank when considering ranksize plots, and much influence classical fits with simple empirical laws (see also a discussion below). For example, the rank-size law of the number $\left(N_{c, r}\right)$ of cities in a given region for the IT regions $(N=20)$ in various recent years is shown in Fig. 2. The data for 2007 and for 2010-2011 are displaced for readability. The fit of $N_{c, r}$, for the (undisplayed) data, with a 3-parameter function

$$
g_{3}(r)=A(N r)^{-\gamma}(N-r+1)^{\beta}
$$

underlines the existence of 3 "regimes": at low, middle and high rank, Fig. 2. Observe that the function presented in Eq. (2.1) generalizes the mere power law $N_{r}=N_{1} r^{-\alpha}$, and takes into account the finite size of the system and the strict vanishing of the distribution at some maximum rank [14-19].

\section{Aggregated tax income}

The analysis of rank-size for ATI confirms a situation appearing also in other rank-size studies of other economic data. On the one hand, it was observed that the distribution of regional ATI values evolved with time $\mathrm{m}$ ore than that of precisely than demographic values [1-4].

On the other hand, looking at the data, after calculating either the number $\left(N_{i, r}\right)$ of inhabitants in a region or the regional ATI $\left(A T I_{r}\right)$, i.e. the ATI sum for the relevant cities in a region, for each year and also for the subsequent quinquennium average, the change in regional membership appeared to be very weakly relevant. Thus, presenting unpublished data, consider the case of the Molise (MOL) region. Molise has $N=136$ cities (and is made of 2 provinces). A rank-size law is hardly represented by a power law (not shown), nor by a simple 2-parameter free function taking into account the finite size of the system, as in

$$
g_{2}(r)=\kappa_{2}\left(\frac{N r}{N-r+1}\right)^{-\chi}
$$

even removing outliers, like the King (Campobasso) and the 3 Vice-Roys (Termoli, Isernia, Venafro) cities in MOL.

These 2-parameter free and 3-parameter free functions (Eqs. (3.1) and (2.1), respectively), for rank-size law research are compared in Fig. 3 and Fig. 4. The various fit coefficients are given in Table I.

It seems, to us and hopefully to the readers, that one can conclude about the necessity of first examining histograms of distributions, during the examination of ranked data, in order to pin point relevant outliers, on 


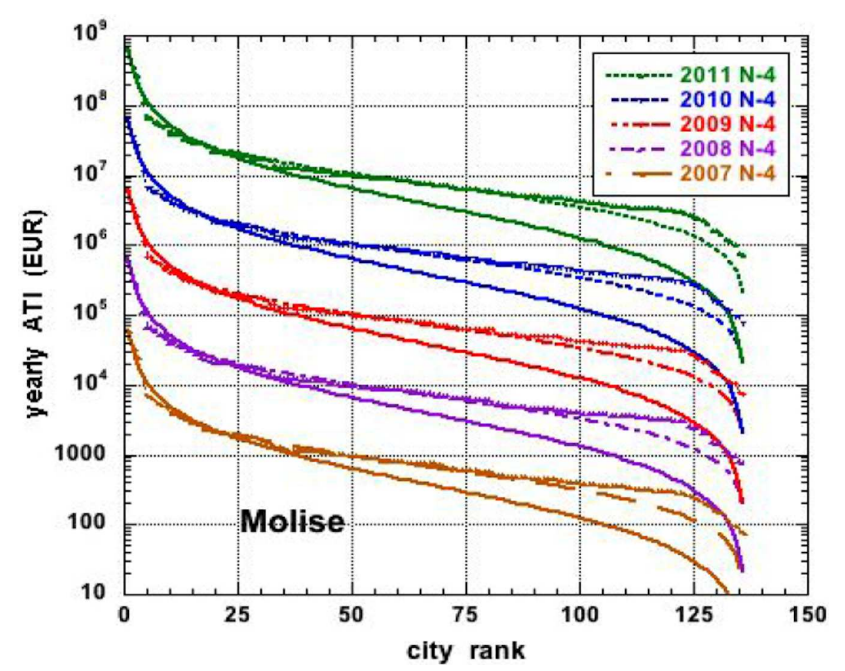

Fig. 3. Displaying the rank-size law of the yearly ATI for Molise, with $N=136$ cities, but after removing the King and 3 Vice-Roy cities, in various recent years: data for 2007-2008 and for 2010-2011 are displaced for readability; fit values for a 2-parameter function, as Eq. (3.1), are given for the (undisplaced) data in Table I.

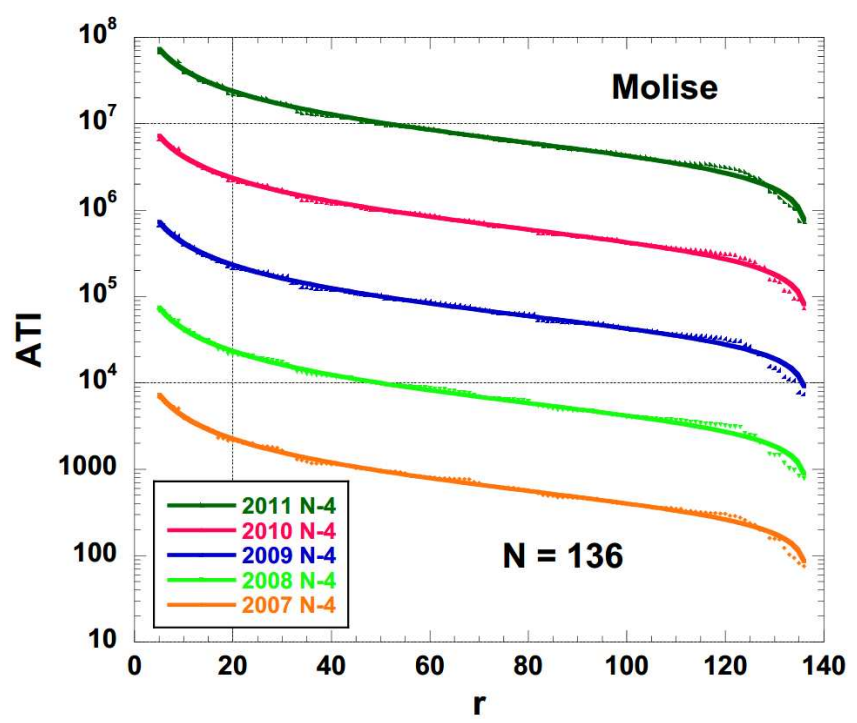

Fig. 4. Displaying the rank-size law of the ATI for Molise, with $N=136$ cities, but after removing the King and 3 Vice-Roy cities, in various recent years: data for 2007-2008 and for 2010-2011 are displaced for readability; fit values for a 3-parameter function, as Eq. (2.1), are given for the (undisplaced) data in Table I.

one hand, and on the other hand, in view of finely reproducing the empirical data through adequate but simple functions.

\section{Financial inequalities}

From the above, it appears that a rank-size law can be useful for examining the evolution of entities in a given ensemble. Other techniques are based on the values leading to a ranking. These techniques are mainly based on a few indicators: the Theil index [7],

\section{TABLE I}

Examples of empirical rank-size law fit: the case of ATI for MOL region with $N=136$ cities, with either 2 or 3 parameters, taking into account all data points but also when excluding the $\mathrm{K}$ and $3 \mathrm{VR}$ cities mentioned in the text. Data are expressed in euros.

\begin{tabular}{|c|c|c|c|c|c|}
\hline \multicolumn{3}{|c|}{ 2-param. } & \multicolumn{3}{|c|}{ 3-param. } \\
\hline & All & -KVR & & All & $-\mathrm{KVR}$ \\
\hline \multicolumn{6}{|c|}{2007} \\
\hline$\kappa_{2}$ & $3.525 \pm 0.26$ & $6.443 \pm 0.113$ & $A$ & $201.0 \pm 251.6$ & $47.090 \pm 9.55$ \\
\hline \multirow{2}{*}{$\chi$} & $1.049 \pm 0.02$ & $0.730 \pm 0.007$ & $\gamma$ & $1.079 \pm 0.02$ & $0.809 \pm 0.01$ \\
\hline & & & $\beta$ & $0.226 \pm 0.25$ & $0.361 \pm 0.04$ \\
\hline$R^{2}$ & 0.979 & 0.990 & $R^{2}$ & 0.979 & 0.993 \\
\hline \multicolumn{6}{|c|}{2008} \\
\hline$\kappa_{2}$ & $3.672 \pm 0.28$ & $6.713 \pm 0.122$ & $A$ & $205.1 \pm 258.4$ & $45.467 \pm 8.41$ \\
\hline \multirow[t]{2}{*}{$\chi$} & $1.046 \pm 0.02$ & $0.726 \pm 0.007$ & $\gamma$ & $1.076 \pm 0.02$ & $0.795 \pm 0.01$ \\
\hline & & & $\beta$ & $0.226 \pm 0.256$ & $0.359 \pm 0.03$ \\
\hline$R^{2}$ & 0.978 & 0.989 & $R^{2}$ & 0.979 & 0.994 \\
\hline \multicolumn{6}{|c|}{2009} \\
\hline$\kappa_{2}$ & $3.539 \pm 0.26$ & $6.842 \pm 0.117$ & $A$ & $318.1 \pm 351.9$ & $38.2595 \pm 7.46$ \\
\hline \multirow[t]{2}{*}{$\chi$} & $1.054 \pm 0.02$ & $0.713 \pm 0.007$ & $\gamma$ & $1.089 \pm 0.02$ & $0.783 \pm 0.01$ \\
\hline & & & $\beta$ & $0.140 \pm 0.225$ & $0.390 \pm 0.04$ \\
\hline$R^{2}$ & 0.979 & 0.990 & $R^{2}$ & 0.980 & 0.993 \\
\hline \multicolumn{6}{|c|}{2010} \\
\hline$\kappa_{2}$ & $3.552 \pm 0.27$ & $6.929 \pm 0.115$ & $A$ & $321.8 \pm 359.6$ & $47.233 \pm 8.93$ \\
\hline \multirow[t]{2}{*}{$\chi$} & $1.053 \pm 0.02$ & $0.709 \pm 0.007$ & $\gamma$ & $1.087 \pm 0.02$ & $0.815 \pm 0.01$ \\
\hline & & & $\beta$ & $0.137 \pm 0.227$ & $0.406 \pm 0.04$ \\
\hline$R^{2}$ & 0.978 & 0.990 & $R^{2}$ & 0.980 & 0.993 \\
\hline \multicolumn{6}{|c|}{2011} \\
\hline$\kappa_{2}$ & $3.605 \pm 0.27$ & $7.031 \pm 0.117$ & $A$ & $432.8 \pm 440.0$ & $35.645 \pm 7.28$ \\
\hline \multirow[t]{2}{*}{$\chi$} & $1.053 \pm 0.02$ & $0.709 \pm 0.007$ & $\gamma$ & $1.086 \pm 0.02$ & $0.780 \pm 0.01$ \\
\hline & & & $\beta$ & $0.141 \pm 0.23$ & $0.406 \pm 0.04$ \\
\hline$R^{2}$ & 0.978 & 0.990 & $R^{2}$ & 0.980 & 0.993 \\
\hline
\end{tabular}

a Herfindahl-Hirschman index [8] and the Gini coefficient [9]. They stemmed from wealth distributions investigations and indicate (financial) inequalities or disparities.

\subsection{Theil index}

The Theil index represents one of the most common statistical tools to measure inequality among data [2021]. Basically it represents a number which synthesizes the degree of dispersion of an agent in a population with respect to a given variable (which plays the role of a measure). The most relevant field of application of the Theil index is represented by the measure of income diversity. The Theil index [7] is defined as:

$$
\mathrm{Th}=-\frac{1}{N} \sum_{i=1}^{N} \frac{y_{i}}{\sum_{j} y_{j}} \ln \left(\frac{y_{i}}{\sum_{j} y_{j}}\right),
$$

where $y_{i}$ is the ATI of the $i$-th city, in our case, and the sum $\sum_{j} y_{j}$ is the aggregation of ATI in regions or in the entire Italy; $N$ is the relevant number of cities when aggregating, according to the $j$ set of interest.

Moreover, it can be easily shown that the Theil index can be expressed in terms of a negative entropy 


$$
H=-\sum_{i}^{N} \frac{y_{i}}{\sum_{j} y_{j}} \ln \left(\frac{y_{i}}{\sum_{j} y_{j}}\right),
$$

where $\frac{y_{i}}{\sum_{j} y_{j}}$ is the "market share" of the $i$-th city, thus indicating a deviation from the "ideal" maximum disorder, $\ln (N)$ :

$$
H=\ln (N)-\text { Th or } \mathrm{Th}=\ln (N)-H .
$$

\subsection{Herfindahl index}

The Herfindahl index, also known as HerfindahlHirschman index (HHI), is a measure of concentration [8]. It is usually applied to describe company sizes (which measure the concentration) with respect to the entire market: a HHI index below 0.01 indicates a highly competitive index (from a portfolio point of view, a low HHI index implies a very diversified portfolio).

Adapted to the case of ATI of cities, HHI is an indicator of the amount of competition (for wealth, here) among municipalities in a region or in the entire country. The higher the value of $\mathrm{HHI}$, the smaller the number of cities with a large value of ATI, the weaker the competition in concurring to the formation of Italian GDP. Formally

$$
H H I=\sum_{i \in L_{50}}\left(\frac{y_{i}}{\sum_{j} y_{j}}\right)^{2},
$$

where $L_{50}$ is the set of the 50 largest cities in terms of ATI, and $y_{i}$ is the ATI of the $i$-th city. The value 50 is conventional, whence HHI in Eq. (4.4) is the sum of the squares of the market shares of the 50 largest cities, when the market shares are expressed as fractions.

A normalized Herfindahl index is sometimes used and defined as:

$$
H^{*}=\frac{(H H I-1 / N)}{1-1 / N} .
$$

with the appropriate $N$.

\subsection{Gini coefficient}

The Gini index $(G i)$ [9] can be viewed as a measure of the level of fairness of a resource distribution among a group of entities. Referring to the specific case treated here, it can be defined through the Lorenz curve, which plots the proportion $f$ of the total Italian ATI that is cumulatively provided by the bottom $x \%$ of the cities. If the Lorenz curve is a line at $45^{\circ}$ in an $f(x)$ plot, then there is perfect equality of ATI. The Gini coefficient is the ratio of the area that lies between the line of equality and the Lorenz curve over the total area under the line of equality. A Gini coefficient of zero, of course, expresses perfect equality, i.e. all ATI values are the same, while a Gini coefficient equal to one (or 100\%) expresses maximal inequality among values, e.g. only one city contributes to the total Italian ATI.

A new type of display is hereby proposed. It seems of interest, for emphasizing the structure (like the maximum position and the corresponding percentage of relevant population) to display the data as the difference between the Lorenz curve $\left(L_{r}\right)$ and the line of perfect equality in ATI:

$$
\Delta L_{j}=\frac{1}{N_{c, r}}\left[\sum_{i=1}^{j} i y_{i, r}-j\right]
$$

with $j \leq N_{c, r}$.

\subsection{Two cases: IT and $M O L$}

As an illustration of the values of such indices, let us give them, and those of the corresponding quantities of interest, in Table II, when calculated for the 5 years of the quinquennium for IT and MOL.

TABLE II

Statistical characteristics of the ATI data distribution as a function of time for the Molise region and for the whole Italy: $N=136$ and 8092, respectively. Entropy is $H$ (see Eq. (4.3)); max. entropy $\equiv \ln (N)$. The Theil index is taken from Eq. (4.1); the Herfindahl index is HHI (see Eq. (4.4)); the normalized Herfindahl index is $H^{*}$ (see Eq. (4.5)).

\begin{tabular}{c|c|c|c|c|c|c}
\hline \hline MOL & 2007 & 2008 & 2009 & 2010 & 2011 & $\langle 5 y a v\rangle$ \\
\hline entropy & 3.6245 & 3.6314 & 3.6371 & 3.6407 & 3.6396 & 3.6350 \\
max. entropy & 4.9127 & 4.9127 & 4.9127 & 4.9127 & 4.9127 & 4.9127 \\
Theil index & 1.2882 & 1.2813 & 1.2756 & 1.2719 & 1.2730 & 1.2777 \\
\hline $10^{2} \mathrm{HHI}$ & 7.6722 & 7.6097 & 7.6336 & 7.6014 & 7.5998 & 7.6225 \\
$10^{2} H^{*}$ & 6.9883 & 6.9253 & 6.9494 & 6.9170 & 6.9153 & 6.9382 \\
\hline Gini coeff. & 0.7007 & 0.6989 & 0.6959 & 0.6957 & 0.6967 & 0.6975 \\
\hline whole IT & 2007 & 2008 & 2009 & 2010 & 2011 & $\langle 5 y a v\rangle$ \\
\hline entropy $(H)$ & 7.2476 & 7.2603 & 7.2659 & 7.2669 & 7.2826 & 7.2650 \\
max. entropy & 8.9986 & 8.9986 & 8.9986 & 8.9986 & 8.9986 & 8.9986 \\
Theil index & $\sim 1.751$ & 1.7383 & 1.7327 & 1.7317 & 1.7160 & 1.7336 \\
\hline $10^{3}$ HHI & 7.332 & 7.236 & 7.205 & 7.230 & 7.115 & 7.222 \\
$10^{3} H^{*}$ & 7.209 & 7.113 & 7.083 & 7.107 & 6.992 & 7.099 \\
\hline Gini coeff. & 0.7591 & 0.7576 & 0.7566 & 0.7565 & 0.7547 & 0.75685
\end{tabular}

It is observed that the yearly variations are rather small. The Theil index is about 1.275. The Gini coefficient is $\approx 0.75$. The HHI is approximately given by $7 \times 10^{-3}$ for IT and $7 \times 10^{-2}$ for MOL. Moreover, in each case, the indices of MOL have a value lower than the corresponding one for IT. The scale effect is the greatest in HHI. Indeed, MOL is known as one of the poorest regions in Italy. Molise is mostly mountainous, and the economy relies heavily on agriculture and livestock raising, although food and garment industries are undergoing some development. The 4 richest cities are markedly dominating in terms of wealth, thereby leading to heterogeneity, reflected in the indices values.

The yearly variation of the Gini coefficient for IT has been presented in [1]. The proposed way, according to Eq. (4.6), is shown in Fig. 5 for IT for the 5 years of interest. It can be observed that the maximum of such a curve occurs at 0.81 of the maximum ATI. The corresponding case for Molise is shown in Fig. 6, but only for the 5 year average data, since it can be imagined that, like for IT, the Gini coefficient of MOL hardly changed during the quinquennium; see Table II for such a justification. However, the maximum of the $\Delta L_{j}$ curve occurs 


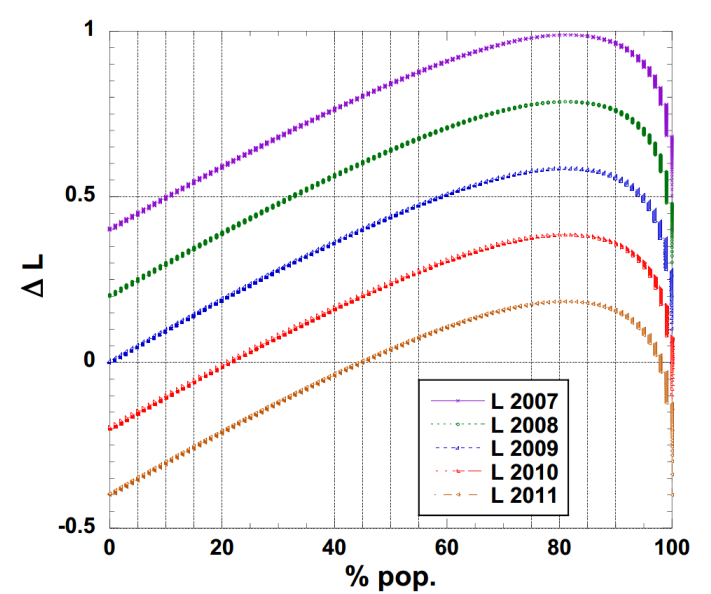

Fig. 5. Another way of representing the Lorenz curve, through Eq. (4.6), leading to the Gini coefficient: the figure presents the case of the cumulated distribution of the AIT in IT 8092 cities during the 5 years of the 20072011 quinquennium; the data (except L009) is displaced for readability.

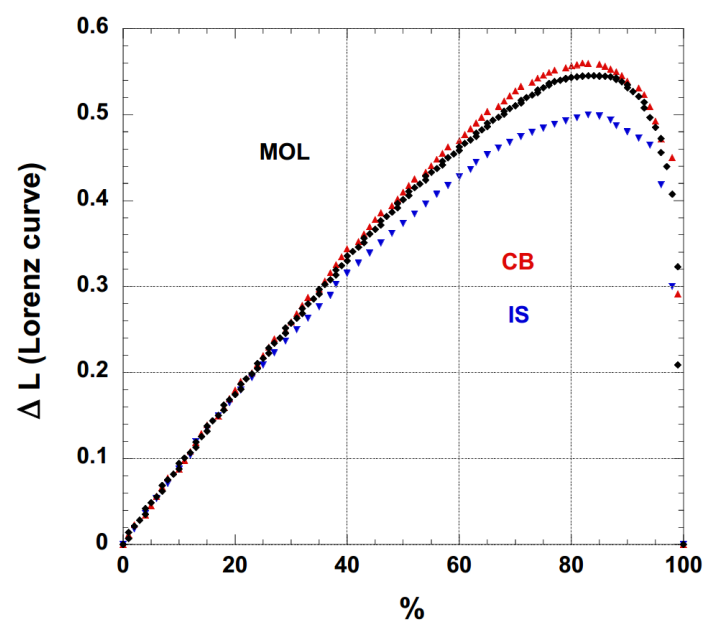

Fig. 6. So called $\Delta$ Lorenz curve, Eq. (4.6), for the cumulated distribution of the AIT in MOL (136 cities), but also for IS (52 cities) and for CB (84 cities) cities during the 5 years of the 2007-2011 quinquennium.

at 0.83 of the maximum ATI, which is after finer examination slightly different from IS and CB, and MOL. It is likely obvious, but a posteriori, that the overall Gini coefficient and $\Delta L_{j}$ curve are much influenced by the larger partner in the examined set, as shown for the case of $\mathrm{CB}$ in MOL in Fig. 6.

\section{Benford law}

Finally, one statistical tool which can serve as a quality/reliability rapid data check is the so-called Benford law $[10,11]$. First digit Benford law for the cumulated distribution of the Molise 186 cities AIT each year of the 2007-2011 quinquennium is shown in Fig. 7: marked deviations are seen, in contrast to other IT regions, as discussed in [6], even though in these some ATI manipulation could have been expected.

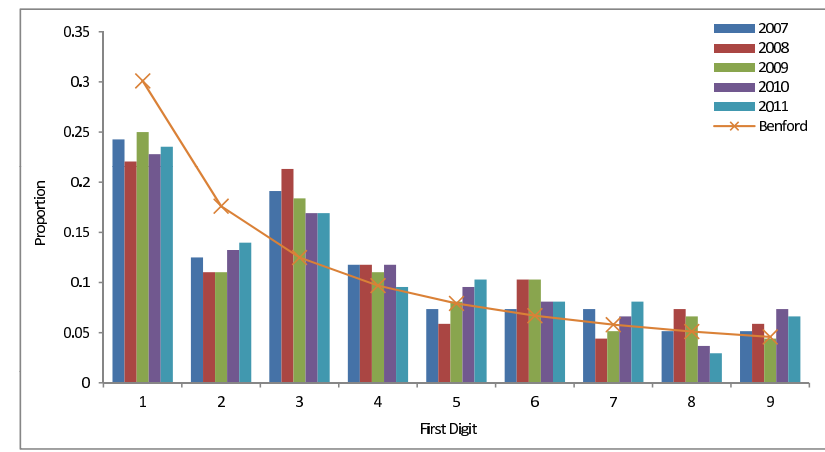

Fig. 7. First digit Benford law for the cumulated distribution of the Molise 186 cities AIT each year of the 2007-2011 quinquennium.

\section{Conclusion}

In the main text, we have applied techniques to investigate regional wealth and administrative (demographically based) disparities through recent ATI data in Italy over the time interval 2007-2011. The rank-size rule is discussed. Yearly data of the aggregated tax income when transformed into the Gini, Theil, and HerfindahlHirschman indices has been analyzed with [22, 23]. One IT region has been selected for illustration and discussion: Molise. A note on the "first digit Benford law" for testing data validity has been presented, indicating an unexpected behavior for the Molise region. Of course, there are other data and means to discuss the wealth of regions, like through measuring territorial dispersion rates of small and medium-sized enterprise creation patterns [24] or as such SME creation related to innovation outputs [25].

\section{Acknowledgments}

MA thanks the FENS 2015 organizers, in particular R. Kutner and D. Grech, for their invitation (and as usual very warm welcome) to the Rzeszów meeting. Thanks to K. Kulakowski for providing extra logistic means. The Benford law plot (Fig. 7) is courtesy of T.A. Mir. Statistical characteristics were obtained due to $[22,23]$.

\section{References}

[1] R. Cerqueti, M. Ausloos, Quality \& Quantity 49, 2307 (2015).

[2] R. Cerqueti, M. Ausloos, Physica A 421, 187 (2015).

[3] R. Cerqueti, M. Ausloos, J. Stat. Mech. 7, P07002 (2015).

[4] R. Cerqueti, M. Ausloos, Soc. Sci. J. 52, 561 (2015).

[5] M. Ausloos and R. Cerqueti, Quality 85 Quantity, in press (July 2015).

[6] T.A. Mir, M. Ausloos, R. Cerqueti, Eur. Phys. J. B 87, 26 (2014).

[7] H. Theil, Economics and Information Theory, Rand McNally and Co., Chicago 1967. 
[8] A.O. Hirschman, Am. Econ. Rev. 54, 761 (1964).

[9] C. Gini, Indici di Concentrazione e di Dipendenza, Biblioteca dell Economista, Serie V, Vol. XX, Utet, Torino 1910 (in Italian); English translation in: Riv. Polit. Econom. 87, 769 (1997).

[10] S. Newcomb, Am. J. Math. 4, 39 (1881).

[11] F. Benford, Proc. Am. Philos. Soc. 78, 551 (1938).

[12] M. Jefferson, Geogr. Rev. 29, 226 (1939).

[13] M. Ausloos, Scientometrics 95, 895 (2013).

[14] I. Popescu, M. Ganciu, M.C. Penache, D. Penache, Rom. Rep. Phys. 49, 3 (1997).

[15] I. Popescu, Glottometrics 6, 83 (2003).

[16] R. Mansilla, E. Köppen, G. Cocho, P. Miramontes, J. Informetr. 1, 155 (2003).

[17] I.A. Voloshynovska, J. Quantitat. Linguist. 18, 274 (2011).
[18] M. Ausloos, J. Appl. Quantitat. Meth. 9, 1 (2014).

[19] J. Miskiewicz, Physica A 387, 6595 (2008).

[20] P. Clippe, M. Ausloos, Physica A 391, 6556 (2012).

[21] M. Ausloos, J. Miskiewicz, Braz. J. Phys. 39, 388 (2009).

[22] P. Wessa, Kendall $\tau$ Rank Correlation (v1.0.11) in Free Statistics Software (v1.1.23-r7), Office for Research Development and Education, 2012.

[23] P. Wessa, Free Statistics Software, Office for Research Development and Education, (v.1.1.23-r7), 2014.

[24] M. Nistotskaya, N. Charron, V. Lapuente, Environm. Plan. C Governm. Policy 33, 1125 (2014).

[25] M. Ausloos, F. Bartolacci, N.G. Castellano, R. Cerqueti, Effects of Innovation on Performance from STAR Market Ranking of Companies, in preparation. 